\section{Active detection of solid-shape information by touch and vision*}

\author{
EUGENE ABRAVANEL $\dagger$ \\ The George Washington University, Washington, D.C. 20006
}

Five groups of Ss were tested under conditions of intra- and intermodal equivalence matching for free-form unfamiliar shapes originally designed by Gibson. Findings indicated that visual intramodal matching was superior to intermodal matching, a result consistent with previous research. The order of accuracy in forming equivalence was: (1) intramodel visual, (2) intramodal haptic, (3) haptic to visual, (4) visual to haptic. A difference, but not a significant one, in accuracy occurred for intramodal haptic matching when Ss wore goggles and when they did not.

Questions regarding the relationship among perceptual systems have ancient roots [Aristotle (McKeon, 1941); Boring, 1942]. Some theorists have emphasized the capacity for intersensory "transfer" and the "formation" of equivalences, using such evidence to argue for a "unity of senses" (Hornbostel, 1938; Werner, 1934). More recently, Gibson (1966) has suggested that the information gathered by one perceptual system is "covariant, coincident, or correlated with the information got by another perceptual system [p. 298]," and that "unity" is achieved in this way. We are now witnessing a revival of interest in the nature of intersensory relationships and a renewed attempt to grapple with fundamental questions such as how unity might be established in terms of biological structures and learning processes (Semmes, Weinstein, Ghent, \& Teuber, 1954; Geschwind, 1965; Abravanel, 1968), the effects of dominance and cooperation between perceptual systems (Rock \& Victor, 1964: Freedman, 1968), and whether or not effective intersensory transfer of training is specifically a human accomplishment (Ettlinger, 1960; Wilson \& Wilson, 1962; Wilson, 1964).

The present investigation derives from a set of root interests similar to those outlined above. Of the possible approaches to the study of intersensory relationships, this research relies on the method of equivalence matching as its tool. To the extent that equivalences can be constructed between two perceptual systems, we may consider the

*This investigation was supported by Biomedical Sciences Support Grant FR-07019-03 from the General Research Support Branch, Division of Research Resources, Bureau of Health Professions Education and Manpower Training, National Institutes of Health.

II would like to express my gratitude to Professor J. J. Gibson of Cornell University for kindly making available the solid shapes used in this study. possibility of some similarity (or unity) of information pickup and processing mechanisms. On the other $h$ and, differences in inter- and intramodal equivalence matching might indicate that information acquisition, coding, and retrieval mechanisms are not fully interchangeable in different modalities.

A number of authors have made a start in the study of intra- and intermodal equivalence matching (Kelvin, 1954; Kelvin \& Mulik, 1958; Caviness, 1964; Rudel \& Teuber, 1964; Krauthamer, 1968; Cashdan, 1968). Shape characteristics as well as size and length dimensions have been studied, but the results have been far from consistent. In some research, intermodal and intramodal matching have been found to be of approximately equal difficulty. Other research has yielded clear results of superior intramodal matching. The bases for the discrepancies are not usually apparent, but new research, profiting from some of the methodological gains of recent work, probably offers the best route to further clarity in this area.

Gibson and Caviness report use of a series of sculptured free-form solid objects designed especially to be appropriate for the study of haptic (active touch) perception (Gibson, 1962, 1966; Caviness, 1964). Each object has a solid three-dimensional shape with five elevations around a central hump. The exact shapes and sizes of the elevated protuberances, as well as the valleys and ridges among them, distinguish each solid from the others in the series. It was judged that these free-form shapes would be useful for further investigation of haptic and visual equivalence matching, as well as for the study of short-term memory demands on mnemonic processes as well as perceptual ones. Transfer of learned events from one system to another implicates memory. Accordingly, the present research was designed to test the following hypotheses: (1) Intramodal shape matching should be more accurate than intermodal matching. (2) Acknowledging the speed and scope of visual shape perception, intramodal visual matching should be highest in accuracy. (3) Haptic intramodal matching should be second in accuracy for the following reasons: the solid objects are appropriate for haptic perception and information pickup; thus, coding and retrieval should be more similar when these processes occur via the same perceptual system. (4) Haptic-visual and visual-haptic (intermodal) matching should be least accurate because a "translation" process (Berkeley, 1709) is required in matching visual shapes across perceptual systems.

\section{SUBJECTS}

The Ss were 125 undergraduate students at The George Washington University, selected primarily from upper-level psychology courses. The Ss had no prior experiences with the Gibson free-form objects and had not previously taken part in any research involving haptic perception. Ss were assigned randomly to one of the three experimental conditions, and to the fourth condition after it was added. Nearly equal numbers of males and females were assigned to each condition.

\section{MATERIALS, APPARATUS,}

AND EXPERIMENTAL SETTING

The stimulus materials were a set of 20 (two matched sets of 10) free-form solid sculptured objects originally used by Gibson (1962) and Caviness (1964). The objects are smooth, black, and of nearly equal size ( $\overline{\mathrm{X}}$ weight $=$ $8 \mathrm{oz}$ ). The rear half of each object is convex, and the front consists of five protuberances around a central hump. Thus, the number of protuberances is the same for all shapes, but the sizes vary, as do the spaces and depths among the protuberances. The entire set consists of 10 identical pairs, each pair different from every other.

Some advantages of these shapes for the comparative study of haptics and vision is that they are solid, unfamiliar, and not easily labeled or associated with familiar objects. Also, their three-dimensional topography lends itself nicely to rapid haptic perception in a way that many outlines do not.

Each object was mounted independently on $5 \times 6$ in. white cardboard so that the distinguishing features of the object could be fully explored.

Comparison pairs for the matching procedure were obtained by having two groups of 10 Ss make 
Table 1

Comparisons of Equivalence Matching Under Intra- and Intermodal Conditions

\begin{tabular}{lcccc} 
& $\begin{array}{c}\text { Total Number } \\
\text { Correct } \\
\text { Matches }\end{array}$ & $\begin{array}{c}\text { Mean } \\
\text { Number } \\
\text { Correct }\end{array}$ & SD & N \\
\hline Visual-Visual & 242 & 9.68 & .47 & 25 \\
Haptic-Haptic II (With Goggles) & 203 & $\mathbf{8 . 1 2}$ & 1.53 & 25 \\
Haptic-Haptic I (Without Goggles) & 190 & 7.40 & 1.32 & 25 \\
Haptic-Visual & 191 & 7.64 & 1.09 & 25 \\
Visual-Haptic & 180 & 7.20 & 1.77 & 25 \\
\hline
\end{tabular}

same-different judgments on 20 pairs of objects. An object was presented for $3 \mathrm{sec}$, followed by a 3 -sec pause, and then a comparison object for $3 \mathrm{sec}$, One group of Ss judged visual pairs, while the second group judged haptic pairs. A decision was made against pairing all objects with each other in order to avoid a too lengthy series.

Through this method, pairs of solid shapes were selected for the main experiment if they were perceived with equal accuracy visually and haptically or with a difference of +1 between modalities ( $\overline{\mathrm{X}}$ visual accuracy $=8.20$ and $\bar{X}$ haptic accuracy $=7.90$ ).

\section{APPARATUS AND}

EXPERIMENTAL SETTING

The $\mathbf{S}$ was seated in a small room $(6 \times 8 \mathrm{ft})$ in front of a table holding the visual screening box. The box (18 $\times 20$ in.) was painted black and contained a shielded hole in the lower center that was large enough for a $S$ to place one hand through but which prevented him from seeing what he was touching. The upper part of the box contained a shelf for placing the visual objects. In order to avoid background visual interference and distraction, a large white curtain was hung from the ceiling to the visual occlusion box, screening the room from wall to wall.

\section{PROCEDURE}

Under all conditions, a modified paired-comparisons procedure was employed. A S received 10 trials, with each trial consisting of: (1) standard object presented for $3 \mathrm{sec}$, (2) followed by a 15-sec interval, and then (3) two comparison objects presented in the same orientation in succession for 3-sec exposures. A 3-sec interval intervened between the first and second comparison object. The intertrial interval was 20 sec.

The S's task was to perceive the shape of the standard object, store this information, then perceive the shape of each comparison object and indicate which of the two comparison objects was the same as the standard. The specifics of procedure for each condition are given below.

Visual-Visual Equivalence

In this condition, for all trials, the standard and two comparisons were presented visually at eye level, approximately $2 \mathrm{ft}$ from the $S$.

\section{Haptic-Visual Equivalence}

The $S$ received each standard object visually for $3 \mathrm{sec}$. Two comparison objects were presented successively for 3 sec of active exploration with the preferred hand (timing commencing when the hand contacted the object). Each comparison object was presented successively for $3 \mathrm{sec}$ of visual inspection.

\section{Visual-Haptic Equivalence}

The $S$ received each standard object visually for 3 sec. Two comparison objects were presented successively for $3 \mathrm{sec}$ of active exploration with the preferred hand.

\section{Haptic-Haptic Equivalence I (Without Goggles)}

Both standard and comparison objects were presented haptically for 3 -sec periods, the $S$ exploring all objects with his preferred hand. The screening device occluded all visual inspection.

\section{Haptic-Haptic Equivalence II}

(With Occluding Goggles)

During the course of administering the three basic conditions, we considered the possibility that intramodal matching under the haptic-haptic condition might have been affected by the fact that Ss performed with eyes open. In order to test this possibility, a further condition was included where Ss performed the haptic-haptic equivalence task while wearing opaque goggles.

\section{PROCEDURE} standard and comparisons, and intertrial time periods were all the same as in previous conditions. The $\mathbf{S}$ explored objects with the preferred hand. In this condition, all Ss were fitted with head goggles that permitted a diffuse yellow-green light but prevented pattern perception.

\section{RESULTS}

Equivalence matching accuracy under intra- and intermodal conditions was significantly different (Table 1).
Object inspection, delay between
Intramodal visual-visual matching was, on average, nearly perfect $(\bar{X}=\mathbf{9 . 6 8})$. Haptic-haptic II (with goggles) matching was second in accuracy $(\overline{\mathrm{X}}=8.12)$. The two intermodal groups achieved lower levels of accuracy: haptic-visual $(\bar{X}=\mathbf{7 . 6 4})$ and visual-haptic $(\bar{X}=7.20)$. Performance in the haptic-haptic I (without goggles) condition $(\overline{\mathrm{X}}=7.40)$ was comparable with that of the intermodal groups.

Analysis of variance across conditions was significant $(F=13.04$, df $=4 / 121, \quad p<.001$ ). Comparing means for the five conditions with the Newman-Keuls procedure (Winer, 1962), the visual-visual condition was significantly different $(p<.01)$ from the means of the other four conditions. No other comparison reached acceptable levels of statistical significance.

Variability was also least under visual-visual matching $S D=.47$ ) and greatest under intermodal visual-haptic matching $(S D=1.77)$.

\section{DISCUSSION}

The findings reported here bear interpretation on several counts. First, insofar as the solid shapes were designed to be appropriate for both visual and haptic perception without any intentional biss toward one modality or the other, we note that, of the two intramodal equivalences, visual matching was more accurate than was haptic matching. Considering that each object was presented for a brief $3 \mathrm{sec}$, the greater speed of visual scanning (and, perhaps, the stronger image created by visual perception) as compared with haptic scanning is probably responsible for the increased efficiency of perception and matching under purely visual conditions. The result partially coincides with that of Caviness (1964), who also found visual discrimination to be somewhat more accurate (but not significantly so) than haptic discrimination with the solid objects used in both of these studies. Two possible bases for the greater difference between visual and haptic intramodal matching found here are (1) that matching was between two successively presented comparisons in the present investigation, while Caviness used a sameness-difference method of matching between pairs of objects, and (2) a 15-sec memory interval transpired between standard object and Comparison 1 in the present investigation, while Caviness had an interval of only 3 sec. Thus, the added importance given to memory functions in the study reported here could account for this discrepancy (as well as for some additional ones to be discussed). With the introduction of a memory factor in equivalence matching, the clarity of the original percept and its resistance to decay or 
interference became increasingly important. This fact probably accounts for the significantly greater accuracy of intramodal visual matching found in this study than in the study by Caviness.

Next, while recognizing the superior accuracy of full visual matching of complex shapes, the accuracy of matching in the haptic-haptic II (with goggles) condition should not be underestimated. An average of fewer than two errors per $S$ was made in this condition. Given the generally uncommon nature of the task where unfamiliar and complex objects must be perceived rapidly by means of touch, an average level of greater than $80 \%$ accuracy is not to be overlooked or misinterpreted. Moreover, we might reasonably expect accuracy of haptic equivalences to approximate visual equivalences with practice.

The similarity of performance for the two intermodal conditions is the third noteworthy finding, but its proper explanation is not readily apparent. In the haptic-visual condition, initial perceptual pickup was accomplished haptically, and the memory trace derived from haptic perception was comparable to that obtained under haptic-haptic matching. Accuracy required proper matching of the haptic trace with the correct visual comparison object in this intermodal condition. The outcome of the process was not significantly different from matching a haptic trace with the correct haptic comparison under haptic-haptic conditions. The findings are similar, although the process may not be, for the other intermodal match, the visual-haptic. Accuracy under visual-haptic and haptic-visual procedures was comparable. Since initial information pickup occurred visually in the visual-haptic condition, the acquisition phase was similar to that for the highly effective visual-visual match. Consequently, the relatively lower accuracy of visual-haptic matching probably occurred at recognition when a visual trace was compared with haptic objects.

Thus, despite similarity of accuracy levels for haptic-visual and visual-haptic matching, there are two different interpretations of process that cannot be disentangled by the present data. The first interpretation is that whenever the standard, the comparisons (or both standard and comparisons) are perceived haptically, accuracy declines relative to intramodal visual matching. The source of greater difficulty may lie in the acquisition, storage, or recognition phases, depending upon whether haptic information is introduced with the standard or the comparisons. Our results will not permit a more precise localization of the difficulty.

The second interpretation deals with the intermodal matches, but in no way applies to the intramodal haptic condition. The notion is that intermodal matching involves a "translation" of haptic impressions into visual impressions, or vice versa. There is also the possibility of verbal mediation playing a part in any "translation" process. Either way, the idea is that in the process of translating or transforming stored information into a form usable for matching with a pair of comparison objects, difficulties arise. Equating shape properties haptically and visually may reduce to a problem of creating the proper "translation rules" between the two modalities.

Each interpretation has certain strengths. The first would account for the lack of a significant difference between intramodal haptic-haptic and intermodal conditions. The second interpretation better elucidates the process that may transpire under intermodal matching, but does not treat the case of intramodal haptic matching. Of course, the lack of a significant difference in accuracies for intermodal and intramodal haptic conditions need not be subsumed under one conceptual umbrella; they may well result from independent factors.

Moreover, the interpretations offered above are most meaningful where shapes are unfamiliar and not easily labeled. Modality effects may not be discernible where objects are familiar or are readily encodable verbally. Also, equivalence matching of unidimensional attributes such as size or length may behave differently (Kelvin, 1954; Kelvin \& Mulik, 1958). To the extent that such attributes are controlled by higher order cognitive operations (e.g., unitizing and composing length), we may find no essential differences between modalities, or between intra- and intermodal functioning (Abravanel, 1971).

Lastly, the finding of somewhat greater success (although not significant) in haptic-haptic matching where Ss were wearing goggles may be methodologically important. Studies of haptic perception, learning, and transfer have used different methods of shielding vision, with screening and blindfolding being the two most common techniques. Comparable results may not always be obtained with different methods, especially where visual competition might be expected during the pickup, encoding, or storage of haptic information.

\section{REFERENCES}

ABRAVANEL, E. The development of intersensory patterning with regard to selected spatial dimensions. Monographs of the Society for Research in Child Development, 1968, 33 (Whole No. 2):

ABRAVANEL, E. The synthesis of length within and between perceptual systems. Perception \& Psychophysics, 1971, 9 327-328.

ARISTOTLE. De Anima. In $R$. McKeon (Ed.), The basic works of Aristotle. New York: Random House, 1941.

BERKELEY, G. An essay towards a new theory of vision. 1709 (any edition).

BORING, E. G. A history of experimental psychology. New York: Appleton, 1942.

CASHDAN, S. Visual and haptic form discrimination under conditions of successive stimulation. Journal of Experimental Psychology, 1968, 76, 215-218.

CAVINESS, J. A. Visual and tactual perception of solid shape. Unpublished doctoral dissertation, Cornell University 1964.

ETTLINGER, G. Cross-modal transfer in monkeys. Behavior, 1960, 16, 56-65.

FREEDMAN, S. J. On the mechanism of perceptual compensation. In $\mathrm{S}$. $\mathrm{J}$. Freedman (Ed.), The neuropsychology of spatially oriented behavior. Homewood, il: Dorsey Press, 1968, $\mathrm{Pp}_{\mathrm{n}}$ 231-240.

GESCHWIND, N. Disconnection syndromes in animals and man. Brain, 1965, 88, 585-644.

GIBSON, J.J. Observations on active touch. Psychological Review, 1962, 69, 477-491. GIBSON, J. J. The senses considered as perceptual systems. New York: Houghton-Mifflin, 1966.

HORNBOSTEL, E. M: von. The unity of the senses. In W. P. Ellis (Ed.), A sourcebook of Gestalt psychology. New York: Harcourt-Brace, 1938.

KELVIN, R. P: Discrimination of size by sight and touch. Quarterly Journal of Experimental Psychology, 1954, 6 . 23-34.

KELVIN, R. P., \& MULIK, A Discrimination of length by sight and touch. Quarterly Journal of Experimental Psychology, 1958, 10, 187-192.

KRAUTHAMER, G. Form perception across sensory modalities. Neuropsychologia, 1968, 6, 105-114.

ROCK, I., \& VICTOR, J. Vision and touch: An experimentally created conflict between the two senses. Science, 1964, 143, 594-596.

RUDEL, R. G., \& TEUBER, H. L. Crossmodal transfer of shape discrimination by children. Neuropsy chologia, 1964, 1, 1-8.

SEMMES, J., WEINSTEIN, S., GHENT, L. \& TEUBER, $H$. $L_{\text {. Performance on }}$ complex tactual tasks after brain injury in man: Analysis by locus of lesion. American Journal of Psychology, 1954, 67, 220-240.

WERNER, H. L'unité des sens. Journal de Psy chologie, 1934, 31, 190-205.

WILSON, M. Further analysis of intersensory facilitation of learning sets in monkeys. Perceptual \& Motor Skills, $1964,18,917-920$.

WILSON, M.. \& WILSON, W. A. Intersensory facilitation of learning sets in normal and brain operated monkeys. Journal of Comparative \& Physiological Psychology, 1962, 55, 931-934.

(Received for publication June $9,1970$. ) 